\title{
ASPECTOS CLÍNICOS E ULTRA-SONOGRÁFICOS DE PACIENTES COM CÂNCER E SUSPEITA DE TROMBOSE VENOSA PROFUNDA
}

\author{
Marcela Pecora Cohen, Julian Catalan, André Piovesan, Rubens Chojniak, Auro Del Giglio*
}

Trabalho realizado no Hospital do Câncer AC Camargo, São Paulo, SP

*Correspondência:

Av. Rebouças, 3357

05410-040, São Paulo, SP

\begin{abstract}
RESUMO
OBJEtivo. Avaliar as características clínicas e evolução dos pacientes com câncer e suspeita de TVP submetidos à USD e a sua sensibilidade.

Métodos. Estudamos 96 pacientes. A mediana da idade foi de 58 anos, sendo que 73 eram do sexo feminino. A USD foi realizada pelo mesmo radiologista em equipamentos digitais (logiq-500-GE e HDI5000-Phillips), com transdutores lineares de alta frequêencia e, quando indicado, convexo de $3,5 \mathrm{mHz}$, utilizando-se modo B e Doppler.

Resultados. Sessenta e sete pacientes $(69,8 \%)$ evoluíram a óbito, sendo que apenas quatro (4,1\%) tiveram exame negativo para TVP e a causa do óbito relacionado a TEP. Quarenta e dois pacientes (43,8\%) não apresentavam TVP à USD, enquanto $33(34,4 \%)$ apresentaram-na, sendo que os demais $(21,8 \%)$ tinham TVP antiga ou trombose superficial. Considerando-se a evolução como padrão ouro e qualquer diagnóstico de TEP desses pacientes causado por TVP, a sensibilidade da USD foi de 95,9\%. Encontramos correlação estatisticamente significativa entre a presença de TVP e o estadiamento da neoplasia ao diagnóstico $(p<0,05)$ e com a sobrevida dos pacientes $(p<0,05)$. Não encontramos correlações significativas com as demais variáveis estudadas.

Conclusã̃o. A USD tem alta sensibilidade para diagnóstico de TVP em pacientes com câncer. A presença de TVP nessa população de pacientes se correlaciona com uma menor sobrevida e não há características clínicas que nos permitam isolar um subgrupo de pacientes com maior chance de TVP por USD.
\end{abstract}

UnITERMOs: Trombose de veias profundas. Ultra-sonografia, diagnóstico e etiologia. Neoplasias - complicações. Fatores de risco - uso diagnóstico.

\section{INTRODUÇÃo}

A associação entre doenças neoplásicas e fenômenos tromboembólicos foi descrita inicialmente por Trousseau em 1865', porém ainda hoje sua fisiopatologia não é clara. Fatores pró-trombóticos liberados pelas próprias células tumorais, elevação de fatores séricos da coagulação ou redução de fatores anticoagulantes naturais por quimioterápicos são alguns dos fatores aventados para explicar tal associação 2.3 .

A real incidência de trombose venosa profunda (TVP) e fenômenos tromboembólicos em pacientes oncológicos varia bastante na literatura (entre II\% e 25\%), de acordo com a série de estudada ${ }^{4}$ e com os fatores agravantes associados, como uso de quimioterápicos (especialmente $\circ$ 5-Fluoracil $)^{5}$, a presença de cateteres intravasculares, cirurgias de grande porte, imobilização, etc ${ }^{6,7}$.

O ultra-som com Doppler (USD) é atualmente o método de escolha para avaliação de trombose no sistema venoso profundo, com sensibilidade e especificidade altas (acima de 95\%), e especialmente para avaliação de pacientes com sintomatologia e TVP de segmentos proximais. $\mathrm{O}$ exame tem baixo custo e permite o diagnóstico diferencial com outras lesões que levam a dor e aumento de volume dos membros inferiores, como cisto de Baker (roto ou intacto), hematoma ou outras massas localizadas, flebite superficial, aneurisma e pseudo-aneurisma arterial; e ainda pode ser realizado à beira do leito em pacientes em estado clínico crítico ${ }^{2-8}$. Entretanto, é um exame cuja acurácia depende do operador e do próprio paciente. Em pacientes obesos, com edema extenso e portadores de variações anatômicas do sistema venoso da região estudada, a avaliação por meio deste exame complementar pode ser prejudicada?.

A complicação mais temida da TVP é o trombo-embolismo pulmonar (TEP), caracterizado por liberação do trombo da luz do vaso afetado, com progressão até as artérias pulmonares e de evolução potencialmente fatal. O TEP também tem forte associação com neoplasias ${ }^{7}$. De fato, em pacientes portadores de neoplasias malignas, a embolia pulmonar é causa comum de óbito (cerca de 7\%) e de severa deterioração do quadro clínico ${ }^{10}$. Portanto, é imperativo o diagnóstico precoce e preciso do quadro de TVP para a implantação de medidas terapêuticas necessárias a fim de evitar o TEP. Muitas instituições usam o USD como método de estudo inicial na pesquisa de TVP em casos de suspeita clínica de TEP por sua elevada acurácia e natureza não- invasiva 8 .

Levando-se em consideração que em pacientes oncológicos há um maior risco de desenvolvimento de TVP/TEP 3,7 e que a USD é um método de alta acurácia e pouco invasivo na pesquisa das complicações trombo-embólicas desses pacientes, o objetivo deste trabalho é avaliar as 
características clínicas e evolução dos pacientes com câncer e suspeita clínica de TVP encaminhados para o setor de ultra-sonografia de um hospital oncológico, para avaliação venosa ultra-sonográfica com Doppler (USD) e o impacto da USD na avaliação desses pacientes.

\section{Métodos}

Avaliamos 98 pacientes com história de neoplasia maligna, em seguimento no Hospital do Câncer AC Camargo, encaminhados ao Departamento de Imagem do mesmo hospital para realização de USD para avaliação de TVP, entre maio de 2001 e abril de 2002. O grupo de pacientes analisado envolveu todos aqueles examinados consecutivamente neste período por um dos autores (MPC). O presente estudo foi aprovado pelo Comitê de Ética em Pesquisa do Hospital AC Camargo.

Os exames foram realizados conforme solicitação do oncologista para pacientes em atendimento ambulatorial, de emergência ou internados, para avaliação dos membros superiores ou inferiores, uni ou bilateral.

Para a USD foram utilizados equipamentos de ultra-sonografia com Doppler pulsado e colorido (logiq 500 - GE e HDI5000 - ATL - Philips) com transdutor linear de alta resolução $(5 \mathrm{a} 12 \mathrm{mHz})$ e, quando necessário, transdutor convexo com baixas freqüências (2 a $5 \mathrm{mHz}$ ) para o estudo de estruturas profundas em relação à pele (especialmente em pacientes obesos ou com edema).

Os exames de membros inferiores incluíram a avaliação das veias profundas (femorais comum, superficial e profunda, poplitea e veias tibiais posteriores) e da veia safena magna, e os de membros superiores também os sistemas venosos profundo e superficial: veias jugular interna, subclávia, axilar, braquial, baślica e cefálica. Foram avaliados a compressibilidade, calibre e conteúdo dos vasos, e a presença e qualidade de fluxo no interior do vaso ao Doppler colorido e pulsado. A ausência de compressibilidade foi o critério mais importante para 0 diagnóstico de TVP, seguido pela ausência de fluxo ao Doppler.

Foram anotadas as características clínicas (coletadas quando da realização do exame ou na análise do prontuário) como: doença de base, estádio da neoplasia ao diagnóstico e à época do exame, quadro clínico que levou à solicitação do exame, a vigência de tratamento por quimioterapia ou radioterapia e história cirúrgica recente. Foram consideradas vigência de quimioterapia ou radioterapia as sessões realizadas no máximo 30 dias antes do exame USD. E, retrospectivamente, por meio da análise de prontuários, avaliamos a evolução dos pacientes.

Também foram anotados os achados do exame USD quanto à presença e nível da TVP (acima ou abaixo do joelho), presença de trombose superficial, pacientes com trombose antiga e recanalização parcial ou completa, presença de edema de aspecto linfático, e os exames de avaliação prejudicada devido à condição clínica ruim ou extenso edema ou obesidade do paciente.

Os pacientes que evoluíram a óbito em até um mês após o exame foram avaliados em relação à causa do óbito quanto à suspeita de TEP. Também para avaliação de TEP foram analisados retrospectivamente os exames complementares dos pacientes como a cintilografia pulmonar por ventilação/perfusão e/ou tomografia computadorizada, quando solicitados.
Foram considerados falso-negativos para TVP os pacientes com exame USD negativo para TVP e que evoluíram com TEP, confirmado por exames complementares ou como causa do óbito em até um mês após o exame. Esse intervalo foi usado com base na fisiopatologia da evolução da trombose venosa aguda, que na primeira semana após a formação do trombo apresenta maior risco de embolia, já que este não está aderido à parede do vaso 1 .

Os dados foram submetidos à análise estatística por meio dos testes Qui-quadrado ou Exato de Fisher para variáveis categóricas, e à análise de variância (Anova) para variávies quantitativas. Para significância estatística foi considerado $p<0,05$. As características clínicas que apresentaram associação significativa com a presença de TVP pela análise univariada foram submetidas à análise multivariada (análise de regressão logística). A análise de sobrevida foi conduzida pelo método de Kaplan-Meier e teste de Wilcoxon. Para representação gráica da análise de sobrevida, utilizamos a curva de Kaplan-Meier.

O presente estudo foi submetido e aprovado pelo Comitê de Ética do Hospital do Câncer AC Camargo.

\section{Resultados}

Foram examinados 98 pacientes consecutivos para avaliação de trombose venosa pela ultra-sonografia com Doppler entre maio de 200 I e abril de 2002. Dois pacientes foram excluídos do estudo, sendo um (o único) pediátrico, com I ano de idade, e outro com TVP de membro superior e presença de port-au-cath no mesmo membro, o que por si só poderia explicar a presença da TVP.

Foram analisados portando 96 pacientes, 73 femininos e 23 masculinos, entre 24 e 92 anos de idade, com mediana de 58,5 anos. Em 78 pacientes o exame foi de membros inferiores, e em 18 pacientes de membros superiores.

Em relação à doença de base, 24 pacientes apresentavam tumor de mama, 12 do colo do útero, dez do cólon e reto, oito de ovário, seis apresentaram linfoma e carcinoma gástrico, quatro tumor do endométrio e melanoma, três leucemia e tumor de bexiga, dois pacientes tumor de próstata e tireóide, dois apresentavam tumor primário desconhecido e os demais tumor de pâncreas, vulva e carcinoma basocelular de pescoço.

O estadiamento dos pacientes à época do diagnóstico e ao exame ultra-sonográfico com Doppler para pesquisa de TVP estão descritos na Tabela I.

\section{Tabela I - Distribuição dos pacientes em relação ao estadiamento do tumor primário à época do diagnóstico e à época da ultra-sonografia com Doppler (USD) para pesquisa de TVP}

\begin{tabular}{|c|c|c|}
\hline \multirow{2}{*}{ Estadiamento } & $\mathrm{N}^{\circ}$ de pacientes & (\%) \\
\hline & $\overline{\text { Aodiagnóstico }}$ & Ao exame USD \\
\hline Localizado & $56(58,3)$ & $18(18,8)$ \\
\hline Disseminado & $31(32,3)$ & $65(67,7)$ \\
\hline "Em remissão" & 0 & $\|(||, 5)$ \\
\hline Seminformação & $9(9,4)$ & $2(2,1)$ \\
\hline Total & $96(100)$ & $96(100)$ \\
\hline
\end{tabular}


Em relação a quimio e radioterapia, 38 (39,6\%) e 14 (14,6\%) pacientes respectivamente estavam em tratamento à época do exame. Cinco entre 24 pacientes com neoplasia mamária estavam em uso de tamoxifeno à época do exame para pesquisa de TVP, e entre elas apenas duas apresentaram trombose venosa: uma profunda e outra superficial. Vinte e um (28\%) pacientes haviam sido submetidos à cirurgia até um mês antes da USD.

Em relação ao quadro clínico que levantou a suspeita de TVP, 84 (87,5\%) pacientes apresentavam pelo menos um sintoma a saber: dor, edema e/ou empastamento. Três pacientes foram submetidos ao exame para pesquisa de TEP sem sintomas de TVP, três para controle de TVP antiga e em seis pacientes não encontramos dados claros no prontuário sobre a condição clínica que motivou a indicação da USD.

Quarenta e dois pacientes tiveram o exame USD negativo para TVP, 33 positivo, nove pacientes foram examinados para controle de TVP antiga e em quatro pacientes foi observada trombose venosa superficial. Em oito (8,3\%) pacientes, o exame foi considerado inconclusivo devido à dificuldade técnica por obesidade ou edema extenso. A freqüência de TVP em nossos pacientes está descrita na Tabela 2.

Quatro (4,1\%) pacientes tiveram exame negativo para TVP e evoluíram a óbito em menos de um mês após o exame, sendo a causa do óbito relacionada a TEP. Nestes pacientes, consideramos o resultado da USD como falso-negativo. Calculamos, assim, a sensibilidade da USD para diagnóstico de TEP em 95,9\%. Quando avaliamos estes quatro pacientes, notamos que um deles apresentava linfoma não Hodking de glândulas lacrimais (estádio III-b), sem tratamento quimio ou radioterápico e com dor e edema do membro inferior esquerdo. Seu exame foi negativo para TVP e em cinco dias evoluiu a óbito por broncoespasmo grave e insuficiência respiratória. O segundo paciente apresentava neoplasia de próstata estádio IV, em vigência de quimioterapia, com edema e empastamento assimétrico dos membros inferiores. Este paciente morreu um mês e dois dias após o exame com edema agudo de pulmão - provável TEP - e tinha estudo de cintilografia pulmonar com alta probabilidade de TEP. O terceiro paciente apresentava tumor de mama estádio IV e em vigência de quimioterapia, com edema e dor no MIE. Esta paciente evoluiu a óbito um mês e um dia após o exame, com ascite volumosa, carcinomatose peritoneal, dispnéia e TEP. O quarto paciente apresentava tumor de mama estádio IV em vigência de quimioterapia, com edema e empastamento simétrico dos MMII. Evoluiu a óbito cinco dias após o exame por insuficiência respiratória grave e derrame pleural massivo, com suspeita de TEP.

Sessenta e sete pacientes $(69,8 \%)$ foram a óbito entre dois dias e 38 meses após o exame, 26 pacientes $(27,1 \%)$ estavam vivos até pelo menos 26 meses após o exame e três pacientes $(3,1 \%)$ perderam o seguimento 5,10 e 15 meses após o exame. Excluindo-se 21 pacientes com trombose antiga, superficial ou exame de avaliação prejudicada, 75 pacientes tiveram diagnóstico definido pela USD, sendo 42 (56\%) sem e 33 (44\%) com TVP.

Entre 33 pacientes com TVP, 28 evoluíram a óbito, sendo três de causa desconhecida (óbito fora do hospital), três relacionados ao TEP e $22(78,5 \%)$ de causas não relacionadas ao TEP. Entre 42 pacientes sem TVP à USD, 3 I evoluíram a óbito, sendo sete por causa

\section{Tabela 2 - Distribuição dos pacientes em relação à presença e forma de trombose venosa}

\begin{tabular}{lc}
\hline Trombose venosa & $\mathbf{N}^{0}$ de pacientes (\%) \\
\cline { 2 - 2 } Ausência de TVP & $42(43,8)$ \\
Presença de TVP & $33(34,4)$ \\
TVPantiga & $9(9,4)$ \\
Trombosesuperficial & $4(4,2)$ \\
Exame duvidoso & $8(8,3)$ \\
Total & $96(100)$ \\
\hline
\end{tabular}

TVP: trombose venosa profunda

\section{Figura I - Gráfico de sobrevida dos pacientes com e sem TVP - curva de Kaplan-Meier (teste de Wilcoxon, $\mathrm{p}<0,02$ )}

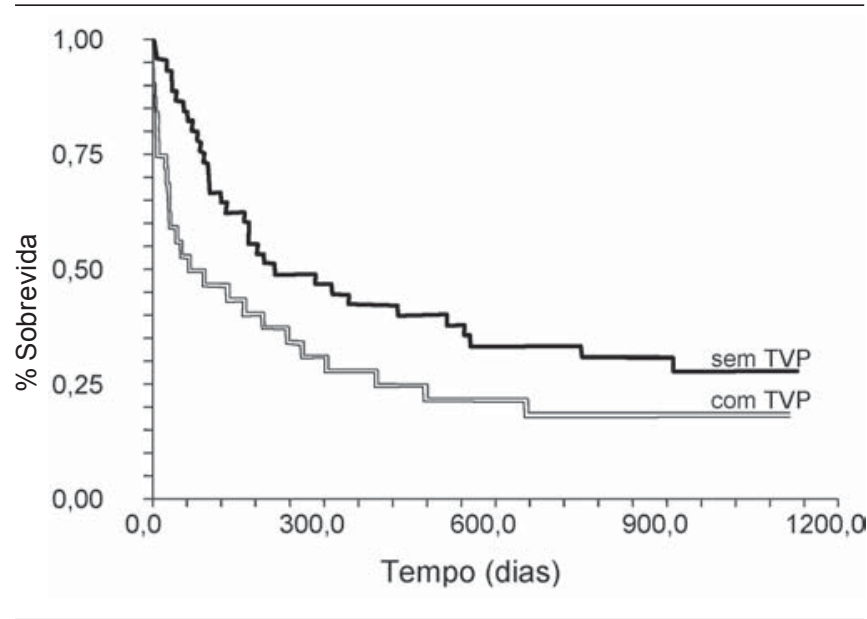

desconhecida, 18 (54,5\%) por causas não ligadas ao TEP e seis por causas ligadas ao TEP em cinco dias (dois pacientes), um mês (dois pacientes), 10 e 18 meses após a USD.

Levando-se em consideração os 75 pacientes com diagnóstico conclusivo de TVP pela USD, não encontramos correlação estatisticamente significativa entre a presença de TVP e os seguintes dados clínicos: sexo, idade, quadro clínico, doença de base, tratamento quimioterápico ou radioterápico, estadiamento clínico por ocasião do exame e a presença de edema linfático.

Encontramos correlação estatisticamente significativa entre a presença de TVP e o estadiamento da neoplasia ao diagnóstico $(p=0,04)$ e com a sobrevida dos pacientes, esta na análise uni $(p=0,02)$ e multi-variada $(p=0,003)$.

\section{Discussão}

A trombose venosa profunda é conhecida há mais de 100 anos como complicação associada ao quadro de malignidade. Estudos populacionais geralmente partem da população geral para avaliar os fatores envolvidos no desenvolvimento da TVP3. Em nosso estudo, partimos da população de pacientes com câncer e suspeita clínica de TVP, encaminhados ao setor de Imagem de um hospital oncológico para avaliação USD para confirmação da TVP. 
Este exame tem sido utilizado rotineiramente como primeiro estudo para pesquisa de TVP, uma vez que é pouco invasivo e apresenta índices de sensibilidade e especificidade altos (95\% e 98\%, respectivamente), para a avaliação do segmento fêmoro-poplíteo, quando comparado com a venografia, que é considerado padrão ouro ${ }^{8}$.

Em nosso estudo, consideramos falso-negativos os exames USD negativos para TVP cujos pacientes evoluíram a óbito em até um mês após o exame, sendo este relacionado ao TEP. Isso ocorreu em quatro $(4,1 \%)$ pacientes, resultando em uma sensibilidade da USD nestes pacientes de 95,9\%. Apesar deste valor estar entre os encontrados na literatura ${ }^{8}$, pode estar subestimado, uma vez que a evolução clínica como "padrão ouro" por nós considerada é bastante rigorosa, já que, mesmo com essa evolução clínica, não sabemos se o trombo que levou ao TEP (que levou ao óbito) teve origem nos membros inferiores, considerando que a freqüência de TVP entre pacientes com TEP confirmada por estudos angiográficos varia entre 10,5\% e |5\% 12,13.

Alguns autores consideram o intervalo de três meses para avaliação do risco de TEP em pacientes avaliados inicialmente com exames negativos para TVP ou TEP'13-16. Esses estudos, porém, não envolvem a população de risco considerada no presente trabalho, mas a populaçãa geral.

Uma vez que a população por nós estudada tem risco maior de desenvolver fenômenos trombo-embólicos do que a população geral ${ }^{3}$ e o risco de embolia pulmonar de origem venosa é maior na primeira semana após a formação do trombo, já que este não está aderido à parede do vaso (fenômeno que ocorre entre a segunda e oitava semana após sua formação)"', foi considerado seguro o seguimento de um mês para relação do TEP com a TVP.

Apesar deste intervalo, considerando o segundo e terceiro mês de evolução na nossa população, mais cinco pacientes com USD negativo para TVP foram a óbito, porém nenhum com causa relacionada a TEP.

As características clínicas de nossa populaçãa em relação ao sexo, idade, quadro clínico, doença de base ou uso recente de quimioterapia ou radioterapia, e a presença de edema linfático, não mostraram correlação significativa com a presença de TVP.

Registramos a presença do edema linfático no intuito de avaliar se, uma vez presente, este pode excluir o diagnóstico de TVP (justificando o edema clínico) ou, ainda, ser responsável pelos exames duvidosos, já que o edema linfático dificulta a visualização e compressão dos vasos profundos e é um achado relativamente comum em pacientes oncológicos. Entre oito pacientes com exame considerado duvidoso, quatro (50\%) apresentavam edema linfático.

O estadio avançado da doença ao diagnóstico e a sobrevida dos pacientes foram as únicas características que apresentaram correlação significativa com a presença de TVP. Podemos interpretar esses achados como: pacientes com estadio avançado de doenças malignas têm maior chance de desenvolver TVP. A pior sobrevida encontrada na nossa população se deveria assim ao estadio avançado, e não necessariamente à TVP, que pode não estar diretamente relacionada ao óbito. Em concordância com nossos achados, Ronsdorf et al. ${ }^{17}$, estudando pacientes com TVP e busca ativa de neoplasia maligna nesses pacientes, encontraram que, na maior parte dos pacientes com TVP e neoplasia, a doença era avançada, e mais da metade desses pacientes evoluíram a óbito em até um ano.
Por outro lado, Sallah et al. ${ }^{7}$, estudando a freqüência de TVP em I04I pacientes portadores de tumores sólidos, não encontraram correlação significativa entre a presença de TVP e a sobrevida dos pacientes, mas encontraram, como nós, associação significativa entre a presença de TVP e o estadio avançado da doença. Para esses autores, o tumor primário mostrou forte associação com a presença de TVP, sendo os mais freqüentes: tumor de células renais, tumor pancreático e gástrico.

Loud et al. ${ }^{2}$ encontraram um número significativamente maior de TVP em pacientes oncológicos e com quadro clínico unilateral (edema e/ou dor assimétricos) do que naqueles pacientes com sintomas bilaterais e simétricos (32\% versus $17 \%$, respectivamente). Entre os nossos pacientes, 68 apresentavam quadro clínico para TVP, sendo assimétrico em 16. Destes, oito (50\%) tinham TVP, dois apresentavam TVP antiga e com recanalização parcial e um, trombose venosa superficial. Os demais não tinham trombose.

$\mathrm{Na}$ análise de nossos dados, não encontramos associação significativa entre a presença de TVP e a doença maligna primária. Atribuímos esta falta de associaçãa com a doença de base ao pequeno número total e ao diagnóstico específico dos pacientes de nossa amostra.

Sabe-se que a presença de neoplasia maligna, por si só, aumenta o risco de desenvolvimento de TVP em até quatro vezes ${ }^{3}$ e a associação com quimioterapia eleva este risco em cerca de seis vezes $s^{3,5,7,10}$. Apesar dessas evidências, não observamos correlação significativa entre a presença de TVP e o uso de quimiterapia ou de radioterapia na nossa população. Isto pode ser explicado pela pequena amostra de pacientes em uso de quimioterapia (39,6\%) ou radioterapia (14,6\%) à época do exame USD para pesquisa de TVP.

\section{Conclusão}

A USD é um exame simples, de baixo custo e alta sensibilidade em pacientes com câncer e sintomas sugestivos de TVP. À exceção de uma correlação significativa do diagnóstico de TVP por USD com menor sobrevida e estadio avançado ao diagnóstico, não encontramos associação com nenhuma outra variável clínica dos pacientes estudados que nos permitisse separar um subgrupo com maior chance de ter TVP diagnosticada por USD. Portanto, acreditamos que o USD deva ser considerado para todos os pacientes oncológicos nos quais há suspeita clínica de TVP.

\section{Conflito de interesse: não há}

\section{SUMMARY}

\section{Prospective evaluation of clinical and Doppler ULTRASOUND CHARACTERISTICS OF CANCER PATIENTS WITH SUSPICION OF DEEP VENOUS THROMBOSIS}

PURPOSE. To evaluate Doppler Ultrasound (DUS) in oncology patients suspected of having Deep Venous Thrombosis (DVT) and correlatefindings with their clinical characteristics and outcome.

METHODS. A study was made with 96 consecutive oncology patients submitted to DUS suspected to have DVT between May 200 I and April 2002. The same radiologist examined 73 women and 23 men, with a median age of 58 years, using DUS equipment, with high frequency linear and convex probes (logic500 - GE and HDI5000 - Philips). 
RESULTS. In 42 (43.8\%) patients the DUS was considered normal, in 33 (34.4\%) DVT was diagnosed and 21 of them had superficial or previous deep venous thrombosis or non conclusive studies. Considering clinical evaluation (and death by PE as caused by DVT) as the gold standard, DUS sensitivity was of $95.9 \%$. There was a statistically significant correlation between DVT and tumor stage at the time of initial diagnosis and patient's survival rate $(p<0.05)$. There was no correlation between DVT and gender, age, type of malignancy, stage of disease at the time of DVT diagnosis and different treatment regimens.

Conclusion. It was concluded that in cancer patients DUS has a high sensitivity for diagnosis of DVT and that DVT diagnosis in these patients correlates with a shorter overall survival. Since there are no reliable clinical predictors to identify cancer patients with DVT, DUS should be performed whenever DVT is suspected. [Rev Assoc Med Bras 2006; 52(5): 360-4]

KEY WORDS: Venous thrombosis - ultrasound, diagnostic, etiology. Neoplasms - complication. Risk factors - diagnostic use.

\section{REFERÊNCIAS}

I. Trousseau A. Phlegmasia Alba dolens. In: Clinique Medical de L' Hotel Dieu de Paris. 2nd ed. Paris: JB Bailliere; 1965. p.654-7I 2.

2. Loud PA, Klippenstein DL. Lower extremity deep venous thrombosis in cancer patients: correlation of presenting symptoms with venous sonographic findings. J Ultrasound Med 1998; 17:693-6.

3. Lee AY, Levine MN. Venous thromboembolism and cancer: risks and outcomes. Circulation 2003; I07(23 Suppl I):117-21.

4. Sciacca FL, Ciusani E, Silvani A, Corsini E, Frigerio S, Pogliani S, et al. Genetic and plasma markers of venous thromboembolism in patients with high grade glioma. Clin Cancer Res 2004; 1 0: 13 12-7.

5. Tham J, Albertsson M.Upper extremity deep venous thrombosis in patients with 5-fluorouracil-containing adjuvant chemotherapy-three case reports and a review. Acta Oncol 2004;43: I 08- 12.

6. Otten HM, Mathijssen J, ten Cate H, Soesan M, Inghels M, Richel DJ, et al. Symptomatic venous thromboembolism in cancer patientes treated with chemotherapy: an underestimated phenomenon. Arch Intern Med 2004; 164:190-4.
7. Sallah S, Wan JY, Nguyen NP. Venous thrombosis in patients with solid tumors: determination of frequency and characteristics. Thromb Haemost 2002;87:575-9.

8. Fraser JD, Anderson DR. Deep venous thrombosis: recent advances and optimal investigation with US. Radiology 1999;21 1:9-24.

9. Quinlan DJ, Alikhan R, Gishen P, Sidhu PS. Variations in lower limb venous anatomy: implications for US diagnosis of deep vein thrombosis. Radiology 2003;228:443-8.

10. Ambrus JL, Ambrus CM, Mink IB, Pickren JW. Causes of death in cancer patients. J Med 1975;6:61-4.

I I. Dupuy DE. 1999 plenary session: friday imaging symposium : venous US of lower-extremity deep venous thrombosis: when is US insufficient? Radiographics. 2000;20: I 195-200

12. Katz DS, Loud PA, Bruce D, Gittleman AM, Mueller R, Klippenstein DL, Grossman ZD. Combined CT venography and pulmonary angiography: a comprehensive review. Radiographics 2002;22:S3-19.

13. Eichinger S, Weltermann A, Minar E, Stain M, Schonauer V, Schneider B, Kyrle PA. Symptomatic pulmonary embolism and the risk of recurrent venous thromboembolism. Arch Intern Med 2004; 164:92-6.

14. Goodman LR, Lipchik RJ, Kuzo RS, Liu Y, McAuliffe TL, O'Brien DJ. Subsequent pulmonary embolism: risk after a negative helical CT pulmonary angiogram-prospective comparison with scintigraphy. Radiology 2000;21 5:535-42.

15. Cornuz J, Pearson SD, Polak JF. Deep venous thrombosis: complete lower extremity venous US evaluation in patients without known risk factors—outcome study. Radiology 1999;21 I:637-41.

16. Ferretti GR, Bosson JL, Buffaz PD, Ayanian D, Pison C, Blanc F, et al. Acute pulmonary embolism: role of helical CT in 164 patients with intermediate probability at ventilation-perfusion scintigraphy and normal results at duplex US of the legs. Radiology 1997;205:453-8.

17. Ronsdorf A, Perruchoud AP, Schoenenberger RA. Search for occult malignancy in patients with deep venous thrombosis. Results of a retrospective cohort study. Swiss Med Wkly 2003; | 33:567-74

Artigo recebido: 04/I 0/2005

Aceito para publicação: 28/04/2006 